\title{
Revue des textes fonciers et forestiers pour la mise en œuvre de la restauration des paysages forestiers à Madagascar
}

\author{
Herimino Manoa Rajaonarivelo!, O. Sarobidy \\ Rakotonarivo', Stefana Raharijaona", Eric Raparison"II, \\ Mirindra Rakotoarisoal, Neal Hockley'v
}

\author{
Correspondance: \\ Herimino Manoa Rajaonarivelo \\ École Supérieure des Sciences Agronomiques \\ Université d'Antananarivo \\ Antananarivo 101, Madagascar \\ Email: manoaherimino@gmail.com
}

\begin{abstract}
RÉSUMÉ
La restauration des paysages forestiers (RPF) vise à restaurer la fonctionnalité du paysage au profit des populations locales, du climat et de la biodiversité. Elle requiert une gouvernance foncière efficace. Cette étude analyse les inconsistances et limites des stratégies nationales et textes juridiques par rapport à l'efficacité de la RPF et avance des recommandations politiques. Notre analyse montre que la loi malgache encourage le reboisement sur terre dégradée dont le domaine de l'État qui n'est pas délimité et souffre d'empiétement. Le succès du reboisement implique la délimitation de ces domaines, l'allègement des procédures pour les acquérir par les communautés locales ainsi que l'inclusion de ces dernières. Une révision des zones forestières sous régime forestier de l'État et des Collectivités Territoriales Décentralisées est aussi requise. Quant aux forêts naturelles, l'incertitude concernant leur statut et les droits des communautés rendent leur restauration très complexe. Nous recommandons que le projet de loi régissant ces terrains à statut spécifique considère plus explicitement la pluralité et la complexité de leur gestion et l'amélioration du droit des communautés locales et du Fokonolona. L'apport de bénéfices concrets aux communautés locales permettrait également de garantir la pérennisation des activités de RPF. Enfin, la restauration effective des zones agroforestières telles que les pâturages est parmi les motifs clés justifient la nécessité de reconnaître légalement et formellement les Fokonolona et les pratiques coutumières. La synergie des cadres juridiques et politiques des différents secteurs est essentielle pour assurer l'efficacité écologique et sociale de la RPF.
\end{abstract}

\section{ABSTRACT}

Forest landscape restoration (FLR) aims to restore landscape functionality for the benefit of people, climate and biodiversity. Increasingly, land tenure and rights are being considered as important enabling factors in FLR. This study analyses tenure considerations in Madagascar's legal texts and FLR policies, outlines their inconsistencies and potential limits in achieving FLR aims, and advances policy recommendations. We found that the current legal texts and framework present several inconsistencies that might impede the effectiveness of FLR in delivering positive environmental and social outcomes. Our analyses demonstrate that most of the target areas for reforestation prescribed by national guidelines are not physically demarcated on the ground and are frequently occupied by local people. This implies that reforestation on degraded lands and its upscaling urgently requires the identification and delimitation of these target lands, and greater involvement of local communities. There are incentives for private individuals or groups to plant trees in return for formalizing their land rights on these target areas, but procedures are very complex, and this has delivered little. To increase the positive impacts on local livelihoods and local uptake, we recommend reducing the complexity of devolving ownership rights of these reforested lands to the local community. A review of forest areas legally vested in the forest regime of the state and decentralized local authorities is also required to promote local participation and reduce potential land conflicts. Regarding natural forests, uncertainties around their legal status and the rights of communities make their restoration very complex. We recommend that the law that is currently being drafted to define the tenure status of these lands explicitly takes into account the plurality and complexity of their management and considers the rights of local communities more explicitly to secure their cooperation. In addition, the sustainability of FLR activities in the long term relies on delivering tangible economic benefits to local communities besides access rights, especially where ecological restoration of native species is prioritized. Currently, these benefits are mostly anticipated from payments for ecosystem services schemes which have not so far materialized. The FLR national strategy and draft law con-

\footnotetext{
I École Supérieure des Sciences Agronomiques, Université d'Antananarivo, Antananarivo 101, Madagascar

II Natural Justice, Lawyers for Communities and the Environment, Mercantile Building, 63 Hout Street, Cape Town, 8000, South Africa

III Solidarité des Intervenants du Foncier - Sehatra Iombonana ho an'ny Fananantany, Lot 149, Cité Ampefiloha, Antananarivo 101, Madagascar

IV College of Environmental Sciences and Engineering, Bangor University, U.K.

Citation Rajaonarivelo, H. M., Rakotonarivo, O. S., Raharijaona, S., Raparison, E., Rakotoarisoa, M. et Hockley, N. 2021. Revue des textes fonciers et forestiers pour la mise en œuvre de la restauration des paysages forestiers à Madagascar. Madagascar Conservation \& Development 16, 1: 32-42.
} http://dx.doi.org/10.4314/mcd.v16i1.4 


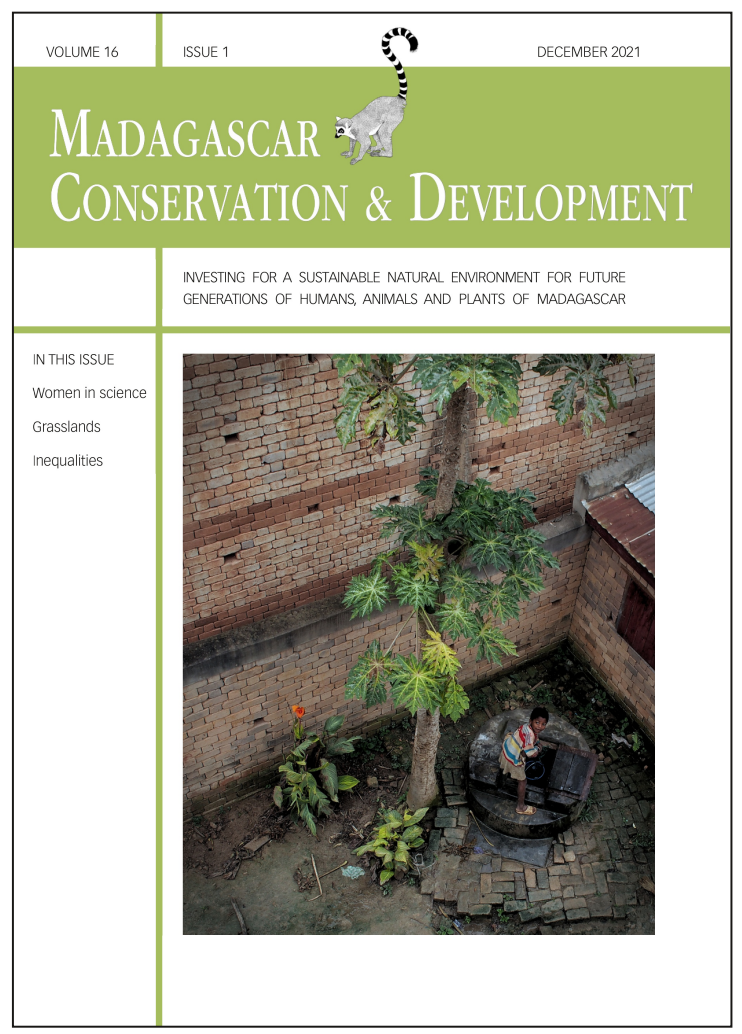

Madagascar Conservation \& Development is the journal of Indian Ocean e-Ink. It is produced under the responsibility of this institution. The views expressed in contributions to MCD are solely those of the authors and not those of the journal editors or the publisher.

All the Issues and articles are freely available at https://www.journalmcd.com

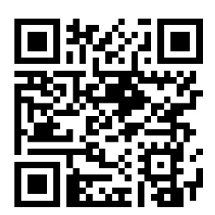

Contact Journal MCD

info@journalmcd.net for general inquiries regarding MCD funding@journalmcd.net to support the journal

Madagascar Conservation \& Development Institute and Museum of Anthropology

University of Zurich

Winterthurerstrasse 190

CH-8057 Zurich

Switzerland

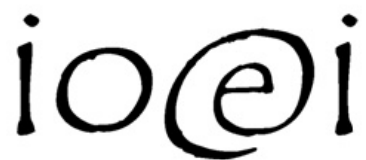

Indian Ocean e-Ink

Promoting African Publishing and Education

www.ioeink.com

Missouri Botanical Garden (MBG)

Madagascar Research and Conservation Program

敨 Missouri Botanical Garden BP3391

Antananarivo, 101, Madagascar 
cerning the restoration of agroforestry areas such as pastures do not currently make any provision for the recognition of the Fokonolona (the village community) or customary practices. This may seriously undermine local communities' rights and risk encouraging land grabbing by more powerful entities. Inconsistencies in legal texts can undermine the effectiveness of the forest restoration in terms of hectares restored, it can also result in poor outcomes for local people. Finally, a greater synergy and coherence of public policies and legal texts across the land management, mining, agricultural and environmental sectors is required to increase the effectiveness of FLR in delivering positive social and environmental outcomes.

\section{INTRODUCTION}

La restauration des paysages forestiers (RPF) suscite de nombreux intérêts du fait des multiples avantages qu'elle offre (Besseau et al. 2018). Elle contribue à la capture du carbone ainsi qu'à la conservation de la biodiversité (Stanturf et al. 2015, Beatty et al. 2018). La restauration des forêts à grande échelle constitue un outil efficace d'atténuation du changement climatique (McCauley et al. 2019). Outre la capacité de la RPF de restauration des fonctionnalités écologiques d'un paysage, cette approche permet aussi d'améliorer le bien-être humain (Stanturf et al. 2017).

La réussite de cette approche repose sur l'implication des communautés locales (Holloway et Short 2014, Erbaugh et al. 2020) et les efforts de restauration peuvent nettement améliorer les conditions de vie locale (Weston et al. 2015, Wilson et Cagalanan 2016). Toutefois, elle risque d'aggraver la pauvreté si sa mise en œuvre restreint l'accès à la terre, aux ressources essentielles et aux moyens de subsistance des populations ou si elle s'accompagne de restrictions liées à la conservation (Adams et Hutton 2007, Rakotonarivo et al. 2017, Poudyal et al. 2018). Le manque de prise en compte des communautés et de leurs droits pourrait entraîner un manque de soutien local et entraver le succès des efforts de restauration (Höhl et al. 2020, Di Sacco et al. 2021).
À Bonn en 2011, Madagascar s'est engagé à relever le défi de restaurer 4 millions d'hectares de paysages et de forêts d'ici 2030 dans le cadre de l'initiative africaine sur la RPF ou AFR100. Cinq types de paysages prioritaires pour la restauration ont ainsi été identifiés: les terres dégradées, les forêts naturelles, les mangroves et les pinèdes dégradées et, enfin, les paysages agroforestiers (République de Madagascar 2017). Toutefois, I'ambiguïté du droit foncier constitue un défi important dans I'usage de la terre et aussi dans l'efficacité de la RPF (République de Madagascar 2017). Une bonne compréhension des règles de gouvernance encadrant I'usage et la gestion des ressources foncières aux niveaux national et local est alors primordiale (Robinson et al. 2018, Mclain et al. 2018).

Cette étude est établie pour analyser les incohérences et limites des textes légaux encadrant l'usage des terres et des forêts ainsi que la gestion des paysages forestiers à Madagascar. Pour ce faire, nous établissons tout d'abord un état des lieux des textes existants se référant à la RPF. Ensuite, nous les analysons et identifions leurs limites. Nous terminons avec des recommandations pour une mise en œuvre plus effective de la RPF.

\section{MÉTHODOLOGIE}

La présente étude s'intéresse aux textes légaux en vigueur liés à la mise en œuvre de la RPF à Madagascar. Ces textes englobent à la fois des textes juridiques tels que les lois, décrets, arrêtés et ordonnances; mais aussi divers instruments tels que les politiques publiques, la vision nationale et les stratégies en vigueur. Les textes sélectionnés soulèvent des questions relatives à la réalisation des activités de RPF. Notre analyse exclut les problèmes liés à l'exploitation forestière qui n'influencent pas directement la mise en œuvre des activités de restauration (Tableau 1).

La majorité des documents a été obtenue en ligne sur le site de la Direction du centre national d'information sur les documentations législatives et juridiques (<http://www.cnlegis.gov.mg/>) des documents a été obtenue auprès des services techniques compé-

Tableau 1. Récapitulatif des textes légaux analysés dans l'étude

\begin{tabular}{|c|c|c|c|}
\hline Type & Date & Secteur & Intitulé / Descriptif \\
\hline Stratégie & 2017 & Environnement & Stratégie nationale sur la restauration des paysages forestiers \\
\hline \multirow[t]{3}{*}{ Vision } & $2019-2023$ & Tous & Initiative émergence à Madagascar \\
\hline & $2015-2019$ & Tous & Plan national de Développement \\
\hline & 2006 & Environnement & Programme d'action national d'adaptation au changement climatique \\
\hline \multirow[t]{9}{*}{ Politique publique } & 2017 & Environnement & Nouvelle politique forestière \\
\hline & 2015-2030 & Energie & Nouvelle politique énergétique \\
\hline & 2015 & Agriculture & Lettre politique agricole \\
\hline & $2015-2030$ & Foncier & Nouvelle lettre politique foncière \\
\hline & $2005-019$ du 17/10/2005 & Foncier & $\begin{array}{l}\text { Fixe les principes régissant les statuts des terres dont le statut foncier spécifique des aires protégées et des } \\
\text { terrains à reboiser }\end{array}$ \\
\hline & 2006-031 du 24/11/2006 & Foncier & Fixant le régime juridique de la propriété privée non titrée \\
\hline & $2008-013$ du 23/07/2008 & Foncier & Régissant le domaine public de l'Etat \\
\hline & 2008-014 du 23/07/2008 & Foncier & Régissant le domaine privé de l'Etat. \\
\hline & $2015-051$ du 03/02/2016 & Aménagement & $\begin{array}{l}\text { Portant sur l'aménagement du territoire ainsi que des dispositions intéressant sur l'aménagement forestier, surtout } \\
\text { sur les réserves foncières forestières }\end{array}$ \\
\hline \multirow{8}{*}{ Loi } & 2015-003 du 19/02/2015 & Environnement & $\begin{array}{l}\text { Charte de l'Environnement malagasy actualisée qui définit des principes généraux de l'environnement en lien avec } \\
\text { le foncier des aires protégées et la politique de reforestation }\end{array}$ \\
\hline & $2015-005$ du 26/02/2015 & Environnement & Refonte du code de gestion des aires protégées \\
\hline & 97-017 du 08/08/1997 & Environnement & Portant révision de la législation forestière \\
\hline & 97-072 du 06/06/1997 & Environnement & Appliquant la sécurisation foncière relative \\
\hline & $96-025$ du 30/09/1996 & Environnement & Relative à la gestion locale des ressources naturelles renouvelables (GELOSE) \\
\hline & 2017-376 du 16/05/2017 & Foncier & $\begin{array}{l}\text { Politique forestière définissant le régime foncier applicable aux aires protégées et aussi le régime de l'utilisation } \\
\text { des terres par les communautés }\end{array}$ \\
\hline & 2017-415 du 30/05/2017 & Environnement & Fixant les modalités et les conditions de la loi n²015-005 sur les codes des AP \\
\hline & 2000-383 du 07/06/2000 & Environnement & Application de l'article 43 de la loi forestière n97-017 \\
\hline \multirow[t]{4}{*}{ Décret } & 2005-849 du 13/12/2005 & Environnement & Portant application de la législation forestière \\
\hline & $2001-122$ du 04/02/2001 & Environnement & Conditions de mise en œuvre de la gestion contractualisée des forêts de l'Etat \\
\hline & $98-610$ du 13/08/1998 & Environnement & Réglementant les modalités de la mise en œuvre de la sécurisation foncière relative \\
\hline & $87-143$ du 20/04/ 1987 & Environnement & Portant des révisions de l'ordonnance 60-127 fixant le régime des défrichements et des feux de végétation \\
\hline Arrêté & $\begin{array}{l}4355 / 1997 \text { du } 13 / 05 / 1997 \\
9398 / 2000 \text { du 05/09/2000 } \\
29211 / 2017 \text { du 28/11/2017 }\end{array}$ & $\begin{array}{l}\text { Environnement } \\
\text { Environnement } \\
\text { Pêche }\end{array}$ & $\begin{array}{l}\text { Portant sur la définition des zones sensibles } \\
\text { Fixant les modalités d'application du décret N } N^{\circ} 2000-383 \text { du 07/06/2000 relatif au reboisement } \\
\text { Fixant les modalités de transfert de gestion des ressources halieutiques et écosvstèmes aquatiques. }\end{array}$ \\
\hline Ordonnance & $\begin{array}{l}\text { 60-127 du 03/10/1960 } \\
75-028 \text { du 22/10/1975 }\end{array}$ & $\begin{array}{l}\text { Environnement } \\
\text { Environnement }\end{array}$ & $\begin{array}{l}\text { Fixant le régime des défrichements et des feux de végétation } \\
\text { Modifiant certaines dispositions de l'ordonnance 60-127 fixant le régime des défrichements et des feux de } \\
\text { végétation. }\end{array}$ \\
\hline
\end{tabular}


tents des ministères concernés. Afin d'analyser les limites des différents textes, des entretiens semi-directifs avec six autorités gouvernementales au sein de ces ministères ont été menés (dont deux du secteur environnement, deux du secteur aménagement du territoire, et deux du secteur foncier). Ces entretiens se sont focalisés sur la praticabilité des textes en vigueur et sur leurs impacts dans la mise en œuvre de la RPF. Par exemple, certaines discussions ont porté sur les textes liés à la sécurisation foncière des domaines forestiers nationaux, la réalisation des restaurations (les zones cibles, les incitations...), la considération des communautés locales et du Fokonolona, les synergies des différentes politiques ministérielles. Ensuite, deux des auteurs (Stefana Raharijaona et Eric Raparison), juristes experts du foncier, nous ont permis de confronter et de mettre à jour les incohérences entre les assertions théoriques et l'application pratique des textes, surtout pour ceux prêtant à confusion et qui pourrait impacter la mise en œuvre de la RPF.

\section{RÉSULTATS ET DISCUSSIONS}

ANALYSE DES CADRES POLITIQUES ET STRATÉGIQUES RELATIFS À LA RPF. Depuis sa participation au défi d'AFR100, Madagascar a élaboré une stratégie nationale de restauration des paysages forestiers (SNRPF). La SNRPF vise à restaurer les paysages forestiers et à les valoriser harmonieusement et durablement pour le bénéfice de la population.

La stratégie est liée aux objectifs de l'Initiative Émergence Madagascar de 2019-2023 qui reconnaît la RPF comme une utilisation pérenne des paysages pour le développement. Outre les actions de lutte contre les feux, les défrichements et la dégradation des aires protégées, cette initiative invoque la nécessité de procéder à une reforestation et à la restauration des forêts, des sols et des bassins versants (République de Madagascar 2018). Cette vision de l'initiative ainsi que la SNRPF s'inscrivent dans la continuité des objectifs du plan national de développement de 2015-2019. Le plan de développement encourage la valorisation durable du paysage par la promotion et le développement des espaces de croissance comme pour les zones spéciales : zones d'investissement agricole et les zones économiques spéciales. Ce plan incite aussi à l'aménagement des bassins versants par le reboisement et la restauration pour un objectif de 35,000 ha par an (République de Madagascar 2015).

Le programme d'action nationale d'adaptation au changement climatique identifie aussi la RPF parmi ses besoins urgents et prioritaires d'actions. Entre autres, elle vise l'adoption de techniques de protection et de restauration du sol, la remise en état des secteurs dégradés par divers reboisements et la promotion des transferts de gestion des forêts (Ministère de l'environnement, des eaux et forêts 2006).

La restauration est tout autant mise en exergue par la nouvelle politique énergétique de 2015-2030. Cette politique insiste aussi sur le besoin de préserver l'environnement tout en valorisant le capital naturel du pays afin de limiter la dépendance au bois et à l'énergie fossile. Elle suggère de protéger les ressources forestières et de réaliser un reboisement de 35000 à 40000 hectares par an afin de sécuriser l'approvisionnement en bois-énergie. Cette politique veut mettre en œuvre un financement national pour le reboisement avec les communautés locales et incite à la délimitation territoriale des zones d'exploitation de bois-énergie (Ministère de I'Energie et des Hydrocarbures 2015).
Les objectifs de la nouvelle politique forestière de 2017 sont aussi cohérents avec la SNRPF. En effet, cette politique a pour objectif de promouvoir le reboisement des terres dégradées et agroforestières. Pour ce faire, elle informe sur le besoin de délimitation consensuelle des terrains à vocation agropastorale et de promotion de la gestion à proximité des ressources naturelles (Ministère de l'Environnement de l'Écologie et des Forêts 2017).

Seule la lettre de politique agricole n'évoque pas la politique de restauration des paysages forestiers. Elle fixe surtout des objectifs dans l'extension des zones agricoles par la mise en place de 1,5 million hectares des zones d'investissement agricole. Ses plans s'attachent à la facilitation d'accès à la terre et à la propriété foncière pour augmenter la surface économique agricole par exploitation (Ministère de l'Agriculture 2015).

Les objectifs de la politique agricole de même que pour la restauration des paysages dépendent de la nouvelle lettre politique foncière de 2015-2030. Elle vise à la facilitation de reconnaissance des parcelles des usagers de terre par la mise en place du Plan d'Occupation Foncière (PLOF) sur tout le territoire dans le cadre de leur mise en sécurité par l'immatriculation. Cela concerne tant les propriétés privées que communautaires. La nouvelle lettre foncière assure la création d'outils de planification de gestion foncière, la précision des limites et usages des aires protégées (AP) et du domaine forestier ainsi que la délimitation des domaines de l'État et des statuts qui englobent toutes les zones spécifiques (Ministère d'État en charge des Projets Présidentiels de l'Aménagement du Territoire et de l'Équipement 2015).

À l'exception de la politique agricole, les cadres politiques en vigueur à Madagascar s'alignent avec l'objectif de la RPF. Ces divers secteurs mettent en exergue le besoin de participer à la restauration des paysages. Ces politiques ne peuvent cependant favoriser la RPF que si la restauration devient la priorité des différents secteurs, notamment sur I'usage des terres qui nécessite un juste équilibre de compromis (Slobodian et al. 2020).

LIMITES JURIDIQUES POUR LE REBOISEMENT SUR TERRES DÉGRADÉES. Difficulté d'identification et de délimitation des terrains de reboisement. En se référant à la loi 2000-383, les réserves foncières pour le reboisement ainsi que les terres sélectionnées par la Commission forestière constituent les zones propices au reboisement. Ces zones peuvent se créer sur des terrains domaniaux (terrains privés ou publics appartenant à l'État), des périmètres de reboisement (terrain destiné au reboisement des personnes privées ou publiques du domaine privé), le domaine forestier national nommé DFN (zones forestières de l'État) et/ou sur les anciennes zones d'action en faveur de l'arbre ou ZODAFARB (domaines privés de l'État délimités pour faciliter l'appropriation du terrain à travers le reboisement pour les reboiseurs en 1984) (art.2). La détermination physique de ces terrains pour le reboisement est assez problématique malgré l'existence de 151 périmètres de reboisement et de restauration anciennement créés sur une superficie totale de 1129372 hectares (FAO 2010). La limite physique de ces zones ne peut être exactement identifiée faute d'immatriculation voire d'absence de décret de création. De plus, avec la réforme foncière de 2005, les zones pouvant accueillir les réserves pour le reboisement sont difficiles à distinguer. La reconnaissance légale des droits coutumiers a presque amené à l'appropriation privative de nombreuses parties des domaines de l'État objet de reboisement. Les incitations foncières au reboisement évoquées à l'article 9 du décret 2000-383 sont ainsi devenues 
inadaptées à cause du pluralisme juridique dans le pays (FAO 2014) qui induit des chevauchements de droits. Le reste des terrains inoccupés appartenant aux domaines de l'État, mais qui n'ont pas fait I'objet d'immatriculation par l'administration, notamment les domaines forestiers incluant les réserves foncières pour le reboisement, font face à un risque d'empiétement (Aubert et al. 2013). De plus, l'inexistence de schéma d'aménagement communal fixant le statut d'usage des terres pénalise la disponibilité de terrain pour la mise en œuvre de reboisement dans la majorité des territoires décentralisés.

Difficulté du processus d'appropriation des terrains reboisés. Les périmètres de reboisement ainsi que des terrains domaniaux affectés au reboisement sont soumis au régime forestier (art. 12 de la loi 97-017). Ils sont ainsi imprescriptibles et inaliénables, mais peuvent néanmoins être accédés à titre individuel par voie de bail emphytéotique (art. 10 du décret n²000-383 et art. 7-12 de son arrêté $\left.n^{\circ} 9398 / 2000\right)$. Ce procédé de bail favorise plutôt le reboisement sur les terrains disponibles des grandes entreprises (Andrianirina Ratsialonana et al. 2010) du fait des clauses à respecter et des coûts d'investissement qui y sont attachés. Pour la motivation des individus ou de la communauté, le cas des terrains domaniaux non affectés ainsi que les anciennes zones d'action en faveur de l'arbre autorisent l'accession à la propriété par les incitations foncières au reboisement (décret n²000-383 et de l'arrêté $n^{\circ} 9398 / 2000$ ). Le processus d'appropriation se base sur le respect des clauses résolutoires rédigées dans un cahier de charge permettant de lever les conditions d'imprescriptibilité pour l'acquisition individuelle de la zone (Ramarolanto-Ratiaray 1989). L'attestation de reboisement ne peut cependant s'obtenir qu'après 5 ans suite à une évaluation positive de la plantation. Cette attestation peut s'achever par une immatriculation sur une période totale de 10 ans à partir de la constatation de la reconnaissance du reboisement de terrain. À cause de la longueur et du coût de ce procédé, les législations forestières en vigueur ne promeuvent pas réellement un support à l'acquisition des terres par le reboisement (Desloges 2001). Les procédures n'ont que rarement abouti à une appropriation légale (Aubert et al. 2015).

Manque de coordination interministérielle. Outre les problèmes d'identification et d'empiétement sur le domaine de l'État, la désignation des autorités responsables en charge de l'identification et de la délimitation des terres destinées au reboisement démontre un conflit interministériel. La loi 2000-071 affirme qu'il revient à la commission forestière de déterminer les zones propices au reboisement. En revanche, l'article 72 de la loi 2015-051 insiste sur le fait qu'il relève des compétences des services techniques chargés de l'aménagement du territoire de déterminer ces zones. On note également un manque de coordination au niveau de l'utilisation de coordonnées géographiques différentes par les diverses entités lors de leur délimitation des terrains identifiés, une problématique régulièrement évoquée. De tels problèmes devraient pourtant être facilement résolus par une conversion et l'unification du type de projection utilisé lors du traitement des images. Néanmoins, le problème d'identification et de protection des zones propices au reboisement persiste faute d'engagement réel de l'État à le dénouer (Aubert et al. 2013) et malgré les nombreuses incitations établies (loi 97-017 art.43, la SNRPF orientation 3 - axe 3, lettre politique foncière orientation 3 - Axe 3.2).

Problème lié à la pérennisation des reboisements. Les reboisements effectués à Madagascar après 1985 se confrontent en majorité à des échecs (Desloges 2001). D'un côté, cet échec s'ex- plique par le manque d'insistance des textes légaux sur la nécessité de maintenir les zones reboisées sous la prescription environnementale, soit à destination forestière ou zone pour plantation d'arbres uniquement. Les clauses régulatrices pour acquérir le terrain suite au reboisement requièrent le respect de la vocation du terrain et des plans de gestion nécessaires (Manjaribe et al. 2013). De même, la loi régissant la propriété privée titrée ou certifiée, requiert cette soumission aux règles d'aménagement prédéfinies pour éviter de renégocier à chaque fois I'application des servitudes forestières et environnementales (République de Madagascar 2017). Une telle pratique, accompagnée du manque d'inclusion des communautés locales dans la planification des reboisements, met en péril la viabilité des plantations d'arbres qui ne sont pas entretenues voire enlevées lorsqu'elles ne répondent pas à leurs besoins (Le et al. 2012). D’un autre côté, la mise en vigueur des textes sur l'usage de feux (décret 87-143 du 20 avril 1987) lors de la pratique de renouvellement de pâturage et de certains feux de végétation sont des pressions permanentes pour les zones restaurées à Madagascar. Les comités responsables du suivi (art. 1 du décret $n^{\circ}$ 2002-793) n'arrivent pas à surveiller l'usage local des feux. De plus, les peines lourdes affligées aux responsables pour réprimander les feux sauvages (ordonnance $n^{\circ}$ 75-028) et pour inciter les collectivités à lutter contre les feux de brousse (décret $n^{\circ}$ 2002-793) ne semblent pas limiter cette pratique. Les feux détruisent ainsi de nombreux hectares de zones reboisées et protégées chaque année (Alvarado et al. 2018) et ont brûlé plus d'un million d'hectares de terres boisées de 2010 à 2014 (FAO 2020).

En tout, malgré les nombreux projets de reboisement vulgarisés depuis longtemps à Madagascar, seule une superficie totale de 312000 ha a réussi à perdurer dans le paysage (FAO 2020). Les limites juridiques précédemment analysées ont en effet contribué à limiter la mise en œuvre des reboisements sur terres dégradées. À petite échelle, I'expansion du reboisement participatif par les communautés locales nécessite l'accès et la sécurité liés à la vaIorisation des terres par les communautés (Desloges 2001, Mclain et al. 2018, Mclain et al. 2019). La mise en œuvre de reboisement à grande échelle convoitée pour atteindre rapidement les bénéfices issus de la RPF (Chazdon 2018) requiert une gouvernance cohérente et une synergie entre les services fonciers et forestiers. En effet, jusque lors, la détermination des terrains de grande superficie sans litige foncier dû au chevauchement de droit coutumier et positif est assez problématique. Les reboisements à grande échelle ont ainsi souvent entraîné la perte d'usage et d'accès des terres (Erbaugh et Oldekop 2018).

INCOHÉRENCES DES TEXTES POUR LA RESTAURATION DE FORÊT NATURELLE. La majorité des forêts naturelles mal-

gaches, à savoir 98\%, relèvent du domaine de l'État (Mansourian et al. 2014). Ces forêts sont soit dans le domaine forestier national (qui est $75 \%$ composé par les stations forestières, les forêts classées et celles dont la gestion a été transférée vers la communauté locale), soit des aires protégées.

Statut et régime juridique flous des aires protégées (AP). La restauration des forêts naturelles se heurte au statut et régime juridique flou de ces zones. Les forêts naturelles sont supposées faire partie du domaine de l'État qui se pose comme seul propriétaire légal (art. 12 de la loi 97-017). Cependant, dans la réalité, les aires protégées incluent une multitude de statuts, surtout au sein des zones périphériques. L'article 3 de la loi 2015-005 attribue le régime juridique des AP suivant leur vocation et les droits existants 
dans ces zones. Suivant ces critères, les noyaux durs des AP relèvent exclusivement du domaine public de l'État ainsi que les réserves naturelles intégrales, au sein desquelles aucune présence ni activité humaine n'est autorisée. Les autres AP (AP publiques dont les parcs nationaux ou naturels, les réserves spéciales outre la réserve naturelle; et les AP mixtes : monuments naturels les paysages harmonieux ainsi que les réserves de ressources naturelles, respectivement de catégorie III et V) peuvent quant à eux contenir plusieurs statuts juridiques car soumis à des régimes de propriété foncière divers. En effet, l'interaction entre homme et nature étant permise dans ces zones ; différentes activités humaines y ont ainsi depuis longtemps eu lieu. La mise en place des AP a par la suite essayé de régir les usages d'un commun accord avec les conventions de gestion communautaire (décret n²017- 415 de la loi 2015-005) mais ces conventions n'existent quasiment pas. La restauration de ces zones s'avère difficile (Elmqvist et al. 2007). Elles ne peuvent être sécurisées du fait de ces incertitudes liées au statut des terres (Mansourian et al. 2016). Elle risque même de porter atteinte aux droits de quelques-uns à cause de la superposition de titres existants dans plusieurs sites protégés (Aubert et al. 2015). La réussite des restaurations à grande échelle des zones forestières par la plantation d'espèces natives dépend cependant de cette prise en compte de I'historique de l'usage du terrain et du contexte de ce paysage (Nunes et al. 2020). Dans le prolongement des dispositions de la loi 2005-019, I'adoption de la nouvelle loi sur les terrains à statuts spécifiques est attendue pour clarifier la situation foncière au sein des aires protégées.

Incertitude des statuts et régimes fonciers des zones restaurées. La détermination des forêts soumises au régime forestier, imprescriptible et insaisissable, génère de la méfiance et nuit à la participation à la restauration de la forêt. Les terrains boisés et reboisés seront assimilés aux forêts sous régime forestier suivant la législation forestière (loi 1997-17). Le problème d'affiliation des zones boisées et reboisées à ce régime peut décourager les participations locales de peur de perdre catégoriquement leurs droits à la terre (Mansourian 2016). D'autre part, même si la terre a eu d'autres vocations que forestière, mais que les régénérations naturelles ou plantées d'arbres couvrent au fur et à mesure la zone, elle sera régie par ce même régime (art.4 de la même loi). La définition juridique des forêts régies sous ces réglementations selon la législation forestière (cf. Matériel supplémentaire) ne contribue pas suffisamment aux objectifs de la restauration forestière. De ce fait, en raison de l'inexistence d'une précision claire des zones sous le régime forestier, la mise en couvert arborée via la restauration d'une zone risque de déroger au droit de propriété en changeant le régime juridique du terrain pour l'État.

Mise à l'écart des droits de propriété de la communauté locale. Des droits communautaires dans le sens d'usage collectif sont reconnus juridiquement dans la législation, mais non le droit de propriété dans le sens du droit coutumier. La loi a en effet des difficultés à reconnaître l'existence de droits de propriété des communautés locales surtout au niveau des terrains à statut spécifique (art 2 de la loi 2006-031). Au sein des forêts naturelles protégées, la loi n'attribue qu'une possibilité de droit de jouissance (Binot et Joiris 2007) aux communautés locales (loi 2015-005 art. 49). Les droits sont restreints aux droits d'usage exercés dans le cadre d'une convention de gestion communautaire. Souvent même dans les pays en développement et y compris à Madagascar, les communautés locales ont été expropriées de leurs terres coutumières pour la conservation des forêts (Oviedo 2005). Les politiques publiques restent aussi muettes quant au droit à la terre des communautés dans les aires protégées, même quand elles participent à des activités de conservation et de restauration.

De même, cette reconnaissance de propriété communautaire est problématique au sein des forêts hors AP définie suivant la loi 97-017 et l'ordonnance 60-127 (les stations forestières, les réserves d'exploitation, les terrains affectés aux services des eaux et forêts et les réserves forestières). Malgré la formulation légale d'une possibilité d'immatriculation grâce à la sécurisation foncière relative de la zone communautaire (art. 15 du décret n 98-610) gérée à travers la gestion locale sécurisée ou GELOSE (loi 96-025, art. 24 de la loi 97-017), seuls les droits de jouissance ont toujours été appliqués. Dans son esprit original, l'esprit de la GELOSE visait pourtant à s'ouvrir à la reconnaissance des propriétés coutumières et leur titrisation (Montagne et al. 2009). L'interprétation de l'administration de cette possibilité de sécurisation comme un mode privilégié d'acquisition de terres après la promulgation des lois foncières de 2005 et 2006, mais aussi l'absence d'un arrêté pour l'application de la sécurisation foncière relative ont alors mis à l'écart les droits de propriété des communautés par les gestionnaires et I'administration. Ce refus de reconnaître I'appropriation communautaire est pourtant la source de nombreuses déforestations mondiales (Zakout et White 2019) menaçant ainsi le maintien de la superficie forestière à Madagascar. Elle limite d'autant plus la participation des communautés aux activités de restauration (Mansourian et al. 2018, Mclain et al. 2019). Le droit réel des communautés locales est alors mis en péril (Aubert et al. 2013) et le problème persiste faute de cadres légaux qui l'explicitent (Aubert et al. 2015). Ce problème entraîne une concurrence dans I'accès, I'occupation et le contrôle de l'espace forestier entre cette communauté et les gestionnaires (Elmqvist et al. 2007). La mise en œuvre de la restauration est alors limitée par ces incohérences des droits des communautés locales (Stanturf et al. 2017). Le refus de l'appropriation de ces terrains par l'administration, sources de subsistance et de bien-être, risque également de dégrader les moyens d'existence des communautés riveraines (Notess et al. 2018).

Absence d'encouragement à la restauration des forêts naturelles. Les cadres politique et juridique à Madagascar intègrent l'importance de la régénération dans la pérennité des forêts pour les générations futures (République de Madagascar 2017). Toutefois, le lien entre communautés et régénération forestière n'est pas solidement établi par les textes. La participation des communautés locales à la régénération forestière n'est pas encouragée et ne donne pas lieu à des avantages concrets. Les seuls avantages accordés à la régénération naturelle des zones dégradées dépendent directement de la nature du projet qui soutient les communautés. Ces avantages pourraient provenir des paiements pour services environnementaux via la REDD+ suite à l'augmentation du stock de carbone forestier par régénération naturelle (objectif 3 de la Stratégie Nationale REDD+). Néanmoins, jusqu'à maintenant, les avantages conséquents attendus de la vente de carbone sont passés inaperçus, car ils se sont réduits à des appuis épisodiques pour les communautés locales (Demaze 2014, Poudyal et al 20018). D'autres avantages plus concrets et pérennes sont ainsi attendus. Par exemple, l'art. 54 de la loi GELOSE a prévu des avantages économiques en guise de compensation d'une bonne gestion de la part des communautés. L'article 54, resté non appliqué (Bertrand et al. 2014, Pollini et al. 2014), devrait pourtant permettre à la communauté de percevoir des bénéfices palpables à travers la parafiscalité qui y est soulevée. Sans la présence de tels avantages répon- 
dant aux besoins locaux, les forêts risquent de disparaître plutôt que d'être gérées durablement (Scales 2014). Les transferts de gestion dont le but unique est la préservation des ressources sans possibilité de valorisation tels que la gestion contractuelle des forêts ou GCF, persistent pourtant encore (Bertrand et al. 2014, Pollini et al. 2014) malgré les expériences positives issues de la valorisation économique durable des ressources menées à Alaotra Mangoro, Boeny et Anosy. Cette stratégie a amélioré la gestion communautaire des forêts grâce à l'autofinancement (contrôle de l'exploitation et de la restauration) ainsi qu'un développement régional (Bertrand et al. 2014). La mauvaise gestion de l'environnement contribuant à un progrès limité de la restauration des forêts persiste alors faute d'une insuffisante application de la loi (Jones et al. 2019)

Manque de synergie dans les gouvernances foncières en relation avec la forêt. Le cadre légal du foncier forestier ne permet pas d'assurer une bonne efficacité de la gestion des forêts. Même si la loi indique que la gestion des forêts relève du ministère chargé de l'environnement, la gestion du terrain n'y est pas incluse. Selon la Lettre Politique Foncière de 2015 (MEATP 2015), la terre est sous la gestion de I'administration foncière. De telles confusions rendent difficile la conservation de la forêt, notamment en cas d'occupation illicite. En effet, malgré leur création, plusieurs AP ne sont pas inscrites dans les PLOF au sein des services de transactions foncières de l'État. De nombreux cas de superposition de titres d'appropriation sont ainsi observés dans plusieurs AP (Aubert et al. 2015). De plus, le plan d'aménagement et de gestion qui détermine les objectifs de gestion des AP et fait partie des outils clés du code des AP (article 25) est absent des dispositions sur les plans de gestion dans la loi 2015-051 en tant qu'outils d'aménagement du territoire. De tels problèmes diminuent le poids et la force de ces plans dans la pratique malgré l'importance de leur contribution dans le cadre de l'aménagement forestier.

INEFFICACITÉ DE LA RESTAURATION DES MANGROVES DÉGRADÉES. Les mangroves font partie du domaine public naturel de l'État (loi 2008-013) et sont classées parmi les zones sensibles (arrêté interministériel n4355/97). Ces forêts publiques offrent une possibilité d'usage individuel ou collectif par l'obtention de permis de coupe (art 40 et 41 de la loi 97-017). En tenant compte de la nature sensible de cet écosystème, les exploitations doivent se soumettre à des exigences strictes (art. 4 du décret 2004-167) telles que le respect des prescriptions d'aménagement établies (art. 10 du décret n98-782). Cet accord d'exploitation est effectué afin d'encourager la participation locale dans la gestion de cette ressource. Cependant, à cause du sous-effectif de l'administration, les contrôles sont quasiment inexistants, laissant à tous un libre accès d'usage (Bertrand et al. 2014, Andriatsiaronandroy 2018). Pourtant, leur conservation voire leur restauration par les communautés locales pourrait avoir des bénéfices concrets, notamment grâce à des financements issus des paiements des services écosystémiques via le REDD+. Le montant obtenu risque de ne pas suffire à la communauté, car l'État reste le bénéficiaire principal en tant que propriétaire (Jones et al. 2016). La forêt de mangrove, au lieu de s'étendre, fait l'objet de déforestation suite aux activités humaines et l'exploitation (Rakotomavo et Fromard 2010). Elle disparaît ainsi d'année en année pour être transformée en charbon, mais aussi pour laisser place à l'extension de l'agriculture ou de l'aquaculture (Giri et Muhlhausen 2008, Jones et al. 2016).

Outre cette problématique, la restauration des mangroves se confronte aux conflits générés par la mise en application de deux différentes procédures de transfert de gestion (GELOSE-GCF face au transfert de gestion des ressources halieutiques (TGRH) de l'ar-

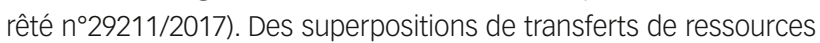
sont en effet observées (c'est le cas de Nosy Be par exemple) entraînant des conflits entre les communautés (la communauté locale de base VOI par rapport aux communautés de pêcheurs par exemple) et sur la gestion des zones transférées (les zones délimitées pour la restauration étant différentes pour le plan d'aménagement et de gestion de la GELOSE/GCF et le plan simple de gestion du TGRH). Cet état indique une inefficacité et incohérence des politiques établies qui affectent de manière importante la conservation et la restauration de ces zones (Mojica Vélez et al. 2018).

LIMITES POUR LA RESTAURATION DES PAYSAGES AGROFORESTIERS. Manque de vision commune entre les politiques des secteurs concernés. À Madagascar, comme ailleurs, I'approche de restauration de ces zones (terrains privés et à statut spécifique comme les pâturages de grande superficie suivant la loi 2008-014) consiste à combiner les plantes agricoles annuelles et les plantes ligneuses pérennes avec des possibilités d'existence de pâturage entre les parcelles (Lacroix et al. 2016). Cependant, les politiques publiques existantes relatives à la forêt, à l'agriculture, au foncier et à l'aménagement ne possèdent pas une telle vision commune. Pour les politiques agricoles et forestières, l'arbre n'est pas considéré dans sa nature multifonctionnelle pouvant contribuer à I'atteinte des objectifs intersectoriels (Saïd et Sibelet 2004). En effet, la politique agricole de 2015 n'inclut pas d'orientation sur l'articulation de l'activité agricole avec la conservation des arbres. La politique forestière quant à elle se concentre plus sur la gestion durable du capital forestier sans prendre en compte l'influence de l'agriculture. La législation forestière semble même au contraire limiter la combinaison des deux pratiques via I'application de son art. 4 qui risque de changer le régime foncier du terrain si l'agroforesterie est pratiquée.

Outre le manque de promotion de l'agroforesterie, aucun plan commun pour assurer la sécurisation des zones de pâturages n'est élaboré par les divers secteurs concernés (fonciers, forestiers, agricoles et d'élevage). Pourtant, inscrire uniquement ces zones dans les plans d'aménagements communaux ne serait pas suffisant du fait du faible pouvoir juridique de ces outils dans la pérennisation de l'usage des terres.

Refus de reconnaissance du Fokonolona. Les paysages agroforestiers constituent 65\% des terres de Madagascar (Dewar et Richard 2012) et assurent la subsistance des communautés ainsi que l'habitat de plusieurs espèces maintenues par les activités pastorales (Solofondranohatra et al. 2020). Dans la pratique, du fait des dispositions coutumières, l'accès et la gestion de ces zones reviennent au Fokonolona, entité de base de la communauté (art. 152 Constitution) et reconnue parmi les acteurs d'aménagement (art. 13 de la loi 2015-051). L'effectivité de la restauration de ces zones est réellement dépendante de leur régime foncier et de leur gouvernance (Robinson et al. 2021). Pourtant, comme de nombreux pays dans le monde (Ykhanbai et al. 2014), l'État malgache ne reconnaît pas le droit d'appropriation de ces zones par cette entité communautaire (Montagne et al. 2009). II a plutôt préféré attribuer la gestion à des groupements de quelques personnes volontaires (dits " communauté locale de base " qui n'est pas tenue d'être représentative de la communauté) qui ne suivent pas le modèle de gouvernance locale basé sur le Fokonolona. Les leçons tirées des 
expériences d'autres régions du monde montrent cependant que les systèmes fonciers rendus incertains ont un impact sur la gestion et la restauration de ces zones gérées par les communautés (Robinson et al. 2021). La reconnaissance du droit foncier communautaire aiderait la communauté à se projeter dans une gestion durable de ces zones et à diminuer le risque d'accaparement des terres (Ykhanbai et al. 2014). À cause du refus de reconnaissance de cette entité et de leur possibilité d'appropriation foncière, la réalisation des délimitations et de sécurisation de ces zones suivant le souhait des politiques publiques (la nouvelle politique forestière et de la lettre politique foncière de 2015) peut affecter négativement le bien-être de la communauté. Les sécurisations peuvent ainsi conduire à un risque de privatisation individuelle de ces zones jusqu'à affecter toute une communauté (Mclain et al. 2019).

\section{RECOMMANDATIONS}

À l'issue de cette revue se dessinent dix recommandations pour permettre une réalisation effective de la RPF à Madagascar.

DANS LE CADRE DU REBOISEMENT DE TERRES DÉGRADÉES.

1. Inventaire parcellaire du domaine privé de l'État: les besoins d'inventorier le domaine de l'État ont déjà été mis en exergue afin de faciliter leur gestion et leur usage. Des expériences des inventaires parcellaires élaborés par certains projets ont montré leur efficacité et pourraient servir de modèle. Par exemple, I'utilisation de la délimitation participative a permis de recenser les divers occupants en impliquant la communauté locale, les autorités traditionnelles, le Fokontany et la Commune (Mclain et al. 2019). Les aînés au sein des communautés locales pourraient s'assurer d'identifier chaque propriétaire coutumier de terrain et les terres sans maître, à justifier par la suite par le Fokontany et la commune. Les services fonciers vérifient à la fin les informations obtenues avec leur base de données avant de classer les zones disponibles dans le domaine de l'État. De telles collaborations des collectivités territoriales décentralisées avec les services topographiques sont également sollicitées lors de la mise en place des PLOF (art L'4 de la loi 2006-031). Lors de la demande d'immatriculation ou d'affectation de la zone, un procès-verbal d'inventaire parcellaire s'avère primordial car cet outil permet d'indiquer la considération de la communauté locale et le respect de leurs droits.

2. Allègement des processus d'acquisition du domaine de l'État : le procédé actuel d'acquisition de terrains via le reboisement limite la motivation individuelle et la réussite du reboisement. Pour pallier à ce problème, des politiques favorables au reboisement et l'apport de bénéfices socio-économiques concrets peuvent aider à accroître l'intérêt des ménages au reboisement (Worku et al. 2018). Entre autres, la révision des cadres légaux sur l'usage de terrains domaniaux par la cessation de terre à reboiser et la délivrance de permis d'utilisation des arbres plantés peuvent augmenter la participation locale au reboisement (Gençay 2020). La réplique des méthodes effectuées par Greenmad (financé par la GIZ) lors de la promotion des reboisements villageois individuels (RVI) est à refléter. Les terrains de l'État détectés sont transférés aux communes et puis affectés à titre individuel à des personnes volontaires au reboisement. Ces zones reboisées font l'objet d'acquisition individuelle après l'évaluation positive de la plantation par rapport au respect du cahier de charge tripartite. De même pour le reboisement effectué lors du Projet d'Appui au Reboisement Villa- geois, les reboiseurs ont pu obtenir des certificats verts à la fin du projet qui représentent un titre de propriété sous prescription environnementale. Les mesures d'obtention du certificat vert avant le titre se ralliaient d'abord au respect des suivis requis lors du reboisement (exécution des travaux d'entretien et de protection du terrain préconisés) (Gabathuler et al. 2014). Répliquer des expériences similaires sur les zones d'action en faveur de l'arbre (ZODAFARB) pour un reboisement communautaire, paraît aussi efficaces car la terre serait déjà transmise à la communauté. La mise en œuvre de reboisements à grande échelle repose sur une combinaison de gouvernance multipartite, incluant tous les secteurs œuvrant au sein du paysage (Erbaugh et Oldekope 2018).

3. Respect des prescriptions d'usage des terrains : les outils cartographiques de planification territoriale (les divers schémas d'aménagement, que ce soit national, régional ou communal, les plans d'urbanisme et les plans d'aménagement et de gestion) devront être réglementés dans leur mise en application afin qu'ils détiennent des forces coercitives sur le respect des prescriptions de terre. La loi doit alors requérir que les activités à mener respectent les outils cartographiques de planification territoriale et le règlement d'aménagement. Dans le cas de zone à vocation forestière destinée au reboisement ou à la restauration, les titres fonciers devraient spécifier de telle prescription pour éviter le changement d'affectation d'usage. La supervision effectuée par les polices d’aménagement de territoire (créées suivant le décret n²017-646) s'avère impérative afin de vérifier le respect d'usage des terrains suivant le texte d'aménagement existant pour assurer l'efficacité de la RPF.

DANS LE CADRE DE LA RESTAURATION DE FORÊTS NATURELLES.

4. Clarification des statuts et régimes des aires protégées et sous droit forestier : pour le cas des AP, les statuts et régimes juridiques flous nécessitent d'être éclaircis pour répondre concrètement aux questions des propriétaires. La loi 2015-005 portant sur les codes des aires protégées devrait être révisée dans cet objectif. Concernant la mise en sécurité des AP ainsi que les autres zones sous droit forestier, les problèmes de procédé d'immatriculation dus aux coûts élevés et la complexité de sa réalisation devraient y être solutionnés. Une des solutions est d'inscrire les AP non publiques dans le domaine privé de l'État et/ou des Communautés territoriales décentralisées (Aubert et al. 2015) pour que l'État se charge de son immatriculation et de les affecter au ministère chargé de l'environnement afin de transférer leur gestion à d'autres entités. L'usage des images satellitaires peut aussi être mobilisé pour permettre la délimitation de ces zones. L'intégration de leurs données numériques dans le plan de repérage des services topographiques territorialement compétents peut à la fois éviter à recourir à l'opération de bornage coûteux et limiter I'octroi de titre privé dans ces espaces protégés.

5. Précision exacte des forêts sous régime forestier : la pérennisation des actions de restauration dépend de la détermination exacte des zones forestières sous régime forestier (imprescriptible et inaliénable). Une telle précision permet de clarifier l'appropriation de certaines zones après leur restauration et reboisement, et ainsi d'accentuer la participation locale. L'enjeu principal de cette définition est aussi de sécuriser les opérations liées aux forêts pour qu'elles ne soient pas perçues comme illégales et arbitraires vis-à- 
vis du droit de propriété. Pour cela, la mise en application du droit forestier sur certaines zones par les commissions forestières doit provenir d'une définition consensuelle de la zone forestière à la fois adaptée aux enjeux diversifiés, aux exigences du moment et en cohérence avec les objectifs. Elle peut contribuer à renforcer le statut des zones dites forestières, de mieux délimiter le domaine privé forestier, de justifier l'application du régime forestier et de sécuriser indirectement les droits des personnes ayant participé aux reboisements.

6. Prise en compte de la complexité des terrains à statut spécifique dans l'élaboration de la loi les régissant : le projet d'élaboration de la loi régissant les zones à statut spécifique (AP, zone sous convention de gestion des ressources naturelles, zone sous droit forestier, zones sous convention internationale) devrait clarifier les questions relatives aux régimes juridiques spécifiques de ces terrains (la mise en articulation entre droits de propriété et le droit d’usage), dans la définition de leurs modalités de reconnaissance, de gestion, de sécurisation, d'acquisition et de perte de droits fonciers. II est requis de prendre en compte les droits des communautés locales sur leurs terres coutumières dont elles ont été les premiers occupants et qui ont été transformés plus tard en aires protégées. Le principal enjeu de cette loi devrait être en outre le développement d'un cadre pour interpréter les dispositions des articles 34 et 152 de la constitution sur la propriété privée et le rôle du Fokonolona dans le développement afin d'aboutir à la reconnaissance juridique de formes de propriété collective.

DANS LE CADRE DE LA RESTAURATION DES PAYSAGES AGROFORESTIERS ET DES FORÊTS NATURELLES.

7. Reconnaissance du Fokonolona et de ses droits fonciers : les textes devraient être révisés de manière à permettre la reconnaissance des Fokonolona et de considérer leurs droits de propriété. La loi devrait consacrer la légitimité des droits fonciers des communautés locales même sous des terrains à statut spécifique en s'inspirant par exemple de la décision sur le peuple endorois du Kenya de la Commission africaine en 2010. Cette décision reconnaît en effet le droit des communautés locales à leur terre ancestrale incluant potentiellement leurs terres coutumières, mais vise aussi à leur protection contre les évictions forcées. Dans le cas d'usage à titre public de ces zones communautaires, des mesures de sauvegarde sociale devraient accompagner les actions menées en guise de compensation des droits locaux. Les mêmes droits devraient aussi s'appliquer dans le cadre des bénéfices obtenus lors des ventes de carbone ou autre. Par exemple, le code des aires protégées (loi 2015-005) reconnaît par ailleurs un droit de propriété aux communautés locales en son article 49 dont la teneur peut être développée par le projet de loi sur les terrains à statut spécifique.

DANS LE CADRE DE LA RESTAURATION DES MANGROVES ET DES FORÊTS NATURELLES.

8. Encouragement à la restauration : d'une part, il faut promouvoir des incitations économiques et/ou foncières pour que la population s'intéresse à la restauration forestière de même qu'au reboisement. De telles initiatives permettent une plus grande participation des communautés et un partage du coût de la restauration. D'autre part, le décret d'application de l'article 54 de la loi GELOSE qui promeut la valorisation durable des ressources naturelles par les communautés comme motivation de la gestion durable desdites ressources devrait être promulgué. De telles valorisations raisonnées des ressources forestières semblent être une solution pour une meilleure gestion des forêts et leur restauration (Bertrand et al. 2014).

POUR TOUTES LES ZONES PRIORITAIRES DE LA RPF À MADAGASCAR.

9. Cohérence et synergie interinstitutionnelle : afin d'assurer la cohérence des activités interinstitutionnelles, la restauration durable des paysages forestiers devrait être effectuée suivant une approche intersectorielle. Les objectifs ministériels devraient alors être voués à l'intérêt général. La RPF doit être imposée telle une vision commune et priorisée dans toutes les activités planifiées de l'État, d'où la nécessité de l'élaboration d'outils de planification pour assurer la bonne gouvernance. Dans ce sens, le Ministère chargé des forêts sur la base des documents nationaux doit élaborer une priorisation claire des attentes en termes d'objectif de restauration pour chaque secteur concerné et des indicateurs d'amélioration de bien-être de la communauté (République de Madagascar 2017).

10. Planification spatiale harmonisée de l'usage des terres: I'efficacité de la RPF s'articule avec une planification spatiale harmonisée par la détermination des zones de reboisement, de restauration des forêts et des paysages agroforestiers. La mise en place d'une telle planification intégrée requiert la coordination de nombreux secteurs concernés (ex. agriculture, pêche et élevage, forêt, foncier, mine) dirigée par celui de l'aménagement du territoire. Les décisions devraient se baser sur une vision nationale qui est de prioriser la restauration des paysages. De ce fait, dans le cadre de la coordination de la déconcentration et de la décentralisation, à partir des schémas d'aménagement national et régional élaborés, les communes devraient être équipées de moyens suffisants pour élaborer un schéma d'aménagement communal pour respecter les diverses prescriptions d'usage de leur zone. Ces schémas devraient ainsi s'assurer de l'articulation entre la planification ayant une vocation écologique avec celle ayant une vocation administrative et socio-économique. La mise en œuvre des schémas d'aménagement communaux ne doit se faire qu'après participation des parties prenantes et du Fokonolona à leur élaboration accompagnée d'un consensus sur leur contenu. Les schémas adoptés doivent revêtir le caractère obligatoire comme prévu par les dispositions de l'article 33 de la loi n²015-05. Ce caractère d'opposabilité aux tiers vise la préservation de l'intérêt général.

\section{CONCLUSION}

La participation de Madagascar au défi de Bonn et de I'AFR 100 souligne la nécessité de restaurer quatre millions d'hectares de paysages forestiers. Le pays possède de nombreux textes relatifs à la mise en œuvre d'un tel objectif qui présentent de nombreuses limites et incohérences. Les analyses des instruments élaborés ont montré que les diverses politiques ministérielles, outre l'agriculture, démontrent l'importance de la restauration. Toutefois, des incohérences entre les textes indiquent un manque de vision commune. La mise en œuvre de la RPF à Madagascar devrait cependant partir de cette base pour acquérir de la cohérence, synergie et harmonie dans l'atteinte globale de ses objectifs. La mise en place de plans d'aménagement décentralisé participatif devrait permettre une planification spatiale harmonisée sur l'ensemble du territoire, ce qui est cruciale pour la RPF. 
Outre les problèmes de synergie et de cohérence entre les ministères, les problèmes fonciers sont une des limites importantes soulevées dans cette analyse. Entre autres, nous avons identifié des difficultés d'identification et de délimitation des domaines de l'État destiné au reboisement, des processus d'appropriation longs et un manque d'insistance des textes sur la pérennisation des terrains reboisés, des statuts et régimes juridiques flous des aires protégées, des incertitudes de statuts des terres des zones restaurées, le refus de reconnaissance des droits fonciers communautaires et de l'entité Fokonolona pour gérer les espaces communs.

Nous recommandons le dénouement de ces problèmes par le biais de la révision et même par l'adoption de nouvelles lois tenant compte de ces problématiques. Par exemple, l'allègement des processus d'acquisition de terre, la clarification des statuts et régimes des aires protégées et la définition des zones sous régime forestier devraient aider à résoudre les problèmes de participation locale. D'autres textes devraient être promulgués, notamment les textes régissant les terrains à statut spécifique ainsi que les textes encourageants à la restauration. Malgré cela, sans considération et participation totale des communautés locales, la mise en œuvre de la RPF présente des risques considérables. II faudrait ainsi réfléchir à la reconnaissance du principe de gouvernance locale par le biais du Fokonolona, et à la reconnaissance des droits communautaires et coutumiers. La collaboration étroite avec les communautés locales a en effet déjà démontré son succès dans la facilitation d'inventaires des domaines de l'État et dans les initiatives de restauration, de suivi et de contrôle des terres restaurées afin d'en assurer la pérennité. Outre les recommandations émises, une meilleure compréhension des perceptions des diverses parties prenantes lors de la conception et de la mise en œuvre des projets RPF s'avère aussi nécessaire.

\section{RÉFÉRENCES}

Adams, W. M. \& Hutton, J. 2007. People, parks and poverty: Political ecology and biodiversity conservation. Conservation and Society 5, 2: 147-183. <https://www.jstor.org/stable/26392879>

Alvarado, S. T., Silva, T. S. F. \& Archibald, S. 2018. Management impacts on fire occurrence: a comparison of fire regimes of African and south American tropical savannas in different protected areas. Journal of Environmental Management 218: 79-87. <https://doi.org/10.1016/j.jenvman.2018.04.004>

Andrianirina Ratsialonana, R., Ramarojohn, L., Burnod, P. et Teyssier, A. 2010. Après Daewoo? États des lieux et perspectives des appropriations foncières à grande échelle à Madagascar. Rome : Observatoire du Foncier à Madagascar, CIRAD, ILC. Accessible en ligne

<https://agritrop.cirad.fr/556661/1/document_556661.pdf>

Andriatsiaronandroy, R. O. 2018. Dynamique récente d'évolution des mangroves de la région de Toliara (Madagascar). Hal Archiv. PhD, Université d'Angers et Université de Tuléar. Accessible en ligne $<$ https://tel.archives-ouvertes.fr/tel-02890385>

Aubert, S., Rambintsaotra, S. et Razafiarijaona, J. 2013. L'insécurité foncière dans et autour des Aires Protégées de Madagascar : un obstacle à surmonter pour la conservation de la biodiversité et le développement rural. Développement Durable et Territoires 4, 1: 1-19.

<https://doi.org/10.4000/developpementdurable.9661>

Aubert, S., Lohanivo, A. C., Rakotondrabe, M., Rahajason, F. et Randriamanarivo N. F. 2015. Analyse de la situation foncière des forêts et de la feuille de route pour la mise en place de la REDD+ à Madagascar. UN-REDD. Accessible en ligne $<$ https://www.unredd.net/documents/redd-papers-and-publications90/15704-analyse-de-la-situation-fonciere-des-forets-et-feuille-de-routepour-la-mise-en-place-de-la-redd-a-madagascar.html>
Beatty, C. R., Cox, N. A. \& Kuzee, M. E. 2018. Biodiversity Guidelines for Forest Landscape Restoration Opportunities Assessments. First edition. IUCN, Gland, Switzerland. Accessible en ligne $<$ https://portals.iucn.org/library/sites/library/files/documents/2018-022En.pdf>

Bertrand, A., Aubert, S., Montagne, P., Lohanivo, A. C. et Razafintsalama, M. H. 2014 Madagascar, politique forestière : Bilan $1990-2013$ et propositions. Madagascar Conservation \& Development 9, 1: 20-30 <https://doi.org/10.4314/mcd.v9i1.4>

Besseau, P., Graham, S. et Christophersen, T. 2018. Restaurer les Paysages Forestiers : la Clé d'un Avenir Durable. Partenariat Mondial pour la Restauration des Paysages Forestiers, Vienne, Autriche. Accessible en ligne $<$ https://www.forestlandscaperestoration.org/images/gpflr_french_final_30jan.pdf>

Binot, A. et Joiris, D. V. 2007. Règles d'accès et gestion des ressources pour les acteurs des périphéries d'aires protégées : Foncier et conservation de la faune en Afrique subtropicale. Vertigo, hors-série 4: 1-17. $<$ https://doi.org/10.4000/vertigo.759>

Chazdon, R. L. 2008. Beyond deforestation: Restoring forests and ecosystem services on degraded lands. Science 320, 80: 1458-1460 <https://doi.org/10.1126/science.1155365>

Demaze, M. T. 2014. L'enrôlement de Madagascar dans la REDD+: domestiquer une opportunité internationale. Vertigo 14,1. <https://doi.org/10.4000/vertigo.14744>

Desloges, V. 2001. Les empreintes de la législation foncière dans l'extension du reboisement à Madagascar. Cahier d'Outre-Mer 54, 213 : 69-94. Accessible en ligne <https://www.persee.fr/doc/caoum_0373-5834_2001_num_54_213_3795>

Dewar, R. E. \& Richard, A. F. 2012. Madagascar: a history of arrivals, what happened, and will happen next. Annual Review of Anthropology 41: 495-517. <https://doi.org/10.1146/annurev-anthro-092611-145758>

Di Sacco, A., Hardwick, K. A., Blakesley, D., Brancalion, P. H. S., Breman, E., et al. 2021 Ten golden rules for reforestation to optimise carbon sequestration, biodiversity recovery and livelihood benefits. Global Change Biology 27, 7: 13281348. <https://doi.org/10.1111/gcb.1549>

Elmqvist, T., Pyykönen, M., Tengö, M., Rakotondrasoa, F., Rabakonandriahina, E. \& Radimilahy, C. 2007. Patterns of loss and regeneration of tropical dry forest in Madagascar: The social institutional context. PLoS ONE 2, 5: e402. <https://doi.org/10.1371/journal.pone.0000402>

Erbaugh, J. T. \& Oldekop, J. A. 2018. Forest landscape restoration for livelihoods and well-being. Environmental Sustainability 32: 76-83. <https://doi.org/10.1016/j.cosust.2018.05.007>

Erbaugh, J. T., Pradhan, N., Adams, J., Oldekop, J. A., Agrawal, A., et al. 2020. Global forest restoration and the importance of prioritizing local communities. Nature Ecology and Evolution 4 : 1472-1476. <https://doi.org/10.1038/s41559-020-01282-2>

FAO. 2010. Rapport National, Madagascar : Programme d'Évaluation des Ressources Forestières Mondiales. ONU. Accessible en ligne $<$ http://www.fao.org/docrep/013/al556f/al556f.pdf>

FAO. 2014. Madagascar et le Pluralisme Juridique : Peut-on Concilier Droit Statutaire et Droit Coutumier pour Promouvoir les Droits Fonciers des Femmes ? Discussion Juridique : synthèse. 14007F/1/08.14. Accessible en ligne <https://www.fao.org/3/a-i4007f.pdf>

FAO. 2020. Évaluation des Ressources Forestières Mondiales 2020. Rapport Madagascar. Accessible en ligne <http://www.fao.org/3/ca9825fr/ca9825fr.pdf>

Gabathuler, E., Ravaoharisoa Rabevohitra, M. V., Rakotondranaly, N. et Bachmann, F. 2014. Reboisements Paysans sur les Hautes Terres Centrales de Madagascar : Capitalisation de Projet de Reboisement Paysan et de ses Impacts après 25 ans. Centre for Development and Environment, Bern, Switzerland. Accessible en ligne <https://boris.unibe.ch/50785/1/REBOISEMENTS.pdf>

Gençay, G. 2020. Legal framework of private afforestation: The case of Turkey. Land Use Policy 96: 104673 <https://dx.doi.org/10.1016/j.landusepol.2020.104673>

Giri, C. \& Muhlhausen, J. 2008. Mangrove forest distributions and dynamics in Madagascar (1975-2005). Sensors 8, 4: 2104-2117. <https://dx.doi.org/10.3390\%2Fs8042104> 
Höhl, M., Ahimbisibwe, V., Stanturf, J. A., Elsasser, P., Kleine, M. \& Bolte, A. 2020. Forest landscape restoration: What generates failure and success? Forests 11, 9 : 938. <https://doi.org/10.3390/f11090938>

Holloway, G. \& Short, S. 2014. Towards a more adaptive co-management of natural resources - increasing social - ecological resilience in southeast Madagascar. Madagascar Conservation \& Development 9, 1: 36-48. <https://doi.org/10.4314/mcd.v9i1.7>

Jones, T. G., Ratsimba, H. R., Carro, A., Ravaoarinorotsihoarana, L., Glass, L., et al. 2016. The mangroves of Ambanja and Ambaro bays, Northwest Madagascar: Historical dynamics, current status and deforestation mitigation Strategy. In: Estuaries: A Lifeline of Ecosystem Services in the Western Indian Ocean. Estuaries of the World. D. S., P. Scheren, J. Ferdinand Machiwa (eds), pp 67-85. Springer, Cham. <https://doi.org/10.1007/978-3-319-25370-1_5>

Jones, J. P. G., Ratsimbazafy, J., Ratsifandrihamanana, A. N., Watson, J. A. M., Andriananadrasana, H. T., et al. 2019. Last chance for Madagascar's biodiversity. Nature Sustainability 2: 350-352. $<$ https://doi.org/10.1038/s41893-019-0288-0>

Lacroix, E., Carodenuto, S., Richter, F., Pistorius, T. et Tennigkeit, T. 2016. Restauration des Paysages Forestiers. Évaluation des Potentialités dans le Contexte des Engagements de Bonn 2.0 et de la Déclaration de New York sur les Forêts. Méthodologie et Résultats pour Madagascar. Unique Forestry and Land Use $\mathrm{GmbH}$, Freiburg, Allemagne. Accessible en ligne $<$ https://afr100.org/sites/default/files/06MDG_RPF_MEOR_Madagascar_Final.pdf>

Le, H. D., Smith, C., Herbohn, J. \& Harrison, S. 2012. More than just trees: Assessing reforestation success in tropical developing countries. Journal of Rural Studies 28, 1: 5-19. <https://doi.org/10.1016/j.jrurstud.2011.07.006>

Manjaribe, C., Frasier, C. L., Rakouth, B. \& Louis, E. E. 2013. Ecological restoration and reforestation of fragmented forests in Kianjavato, Madagascar. International Journal of Ecology, 2: 726275. <https://doi.org/10.1155/2013/726275>

Mansourian, S. 2016. Understanding the relationship between governance and forest landscape restoration. Conservation and Society 14, 3: 267-278. <https://doi.org/10.4103/0972-4923.186830>

Mansourian, S., Aquino, L., Erdmann, T. K. \& Pereira, F. 2014. A comparison of governance challenges in forest restoration in Paraguay's privately-owned forests and Madagascar's co-managed state forests. Forests 5, 4: 763-783. <https://doi.org/10.3390/f5040763>

Mansourian, S., Razafimahatratra, A., Ranjatson, P. \& Rambeloarisao, G. 2016. Novel governance for forest landscape restoration in Fandriana Marolambo, Madagascar. World Development Perspectives 3: 28-31. $<$ https://doi.org/10.1016/j.wdp.2016.11.009>

Mansourian, S., Razafimahatratra, A. \& Vallauri, D. 2018. Lessons Learnt from 13 Years of Restoration in a Moist Tropical Forest: The Fandriana- Marolambo Landscape in Madagascar. WWF, field series 2018. Accessible en ligne <https://www.wwf.fr/sites/default/files/doc-201902/201812_Lessons_Learnt_from_13_Years_of_Restoration_in_a_Moist_Tropi cal_Forest_The_Fandriana_Marolambo_Landscape_in_Madagascar-min.pdf>

McCauley, L. A., Robles, M. D., Woolley, T., Marshall, R. M, Kretchun, A. \& Gori, D. F. 2019. Large-scale forest restoration stabilizes carbon under climate change in the Southwest United States. Ecological Applications 29, 8: e01979. $<$ https://doi.org/10.1002/eap.1979>

Mclain, R., Lawry, S., Guariguata, M. R. \& Reed, J. 2018. Toward a tenure-responsive approach to forest landscape restoration: A proposed tenure diagnostic for assessing restoration opportunities. Land Use Policy 104: 103748. <https://doi.org/10.1016/j.landusepol.2018.11.053>

Mclain, R., Ranjatson, P., Lawry, S., Rakotonirina, J. M. \& Randrianasolo, R. 2019. Tenure challenges to implementing forest landscape restoration in northwestern Madagascar, InfoBriefs CIFOR 273: 1-8. <https://doi.org/10.17528/cifor/007492> <https://doi.org/10.17528/cifor/ 007563>

Ministère d'État en charge des Projets Présidentiels de l'Aménagement du Territoire et de l'Équipement. 2015. Nouvelle Lettre de Politique Foncière. <http://extwprlegs1.fao.org/docs/pdf/mad163912.pdf>

Ministère de I'Agriculture. 2015. Lettre Politique de l'Agriculture. Accessible en ligne <http://extwprlegs1.fao.org/docs/pdf/mad163939.pdf>

Ministère de l'Énergie et des Hydrocarbures. 2015. Lettre de Politique de l'Énergie de Madagascar 2015-2030. Accessible en ligne <http://www.ore.mg/Publication/Rapports/LettreDePolitique.pdf>
Ministère de l'Environnement de l'Écologie et des Forêts. 2017. Politique forestière de Madagascar - Vers une Gestion durable et responsable des Forêts Malagasy. Accessible en ligne <http://faolex.fao.org/docs/pdf/Mag176037.pdf>

Ministère de I'Environnement, des Eaux et des Forêts, Banque Mondiale. 2006. Programme d'Action national d'Adaptation au Changement climatique. Accessible en ligne <https://unfccc.int/resource/docs/napa/mdg01f.pdf>

Mojica Vélez, J. M., Barrasa García, S. \& Espinoza Tenorio, A. 2018. Policies in coastal wetlands: Key challenges. Environmental Science \& Policy 88: 72-82. $<$ https://doi.org/10.1016/J.ENVSCI.2018.06.016>

Montagne, P., Maafaka, R., Aubert, S., Andriambolanoro, D. et Randrianarivelo., G. 2009. La Sécurisation Foncière Relative dans le contexte de réforme foncière à Madagascar : le cas du kijana de Berinrinina. Archive ouverte Hal-CIRAD. Accessible en ligne <http://hal.cirad.fr/cirad-00843856>

Notess, L., Veit, P. G., Monterroso, I., Andiko, Sulle, E., et al. 2018. The Scramble for Land Rights: Reducing Inequity between Communities and Companies. WRI Report. Accessible en ligne $<$ https://www.wri.org/research/scramble-land-rights>

Nunes, S., Gastauer, M., Cavalcante, R. B. L., Ramos, S. J., Caldeira Jr., C. F., et al. 2020 Challenges and opportunities for large-scale reforestation in the Eastern Amazon using native species. Forest Ecology and Management 466: 118120. <https://doi.org/10.1016/j.foreco.2020.118120>

Oviedo, G. 2005. Land ownership and forest restoration. In: Forest Restoration in Landscapes: Beyond Planting Trees. Mansourian, S. Vallauri, D. \& Dudley, N. (Eds.). pp 84-93. Springer, New York. <https://doi.org/10.1007/0-387-29112-1_12>

Pollini, J., Hockley, N., Muttenzer, F. D. \& Ramamonjisoa, B. S. 2014. The transfer of natural resource management rights to local communities. In: Conservation and Environmental Management in Madagascar. Scales, I. R. (ed). pp 172-194. Routledge, London and New York. <https://doi.org/10.4324/9780203118313-20>

Poudyal, M., Jones, J. P. G., Rakotonarivo, O. S., Hockley, N., Gibbons, J. M., et al. 2018 Who bears the cost of forest conservation? PeerJ 7: e5106. $<$ https://doi.org/10.7717/peerj.5106>

Rakotomavo, A. \& Fromard, F. 2010. Dynamics of mangrove forests in the Mangoky River delta, Madagascar, under the influence of natural and human factors. Forest Ecology and Management 259, 6: 1161-1169. <https://doi.org/10.1016/j.foreco.2010.01.002>

Rakotonarivo, O. S., Jacobsen, J. B., Larsen, H. O., Jones, J. P. G., Nielsen, M. R., et al. 2017. Qualitative and quantitative evidence on the true local welfare costs of forest conservation in Madagascar: Are discrete choice experiments a valid ex ante tool? World Development 94 : 478-491. <https://doi.org/10.1016/j.worlddev.2017.02.009>

Ramarolanto Ratiaray 1989. L'accès à la terre en droit rural malgache. Revue Internationale de Droit Comparé 41, 3: 637-707. Accessible en ligne <https://www.persee.fr/doc/ridc_0035-3337_1989_num_41_3_1794>

République de Madagascar. 2015. Plan National de Développement. Accessible en ligne $<$ <ttps://www.pseau.org/outils/ouvrages/ mid_plan_national_de_developpement_de_madagascar_2015_2019_pnd_20 15.pdf>

République de Madagascar. 2017. Stratégie nationale sur la Restauration des Paysages forestiers et des Infrastructures vertes à Madagascar. Accessible en ligne <https://afr100.org/sites/default/files/07-RPF_MDG_SNRPF.pdf>

République de Madagascar. 2018. Initiative Émergence Madagascar. Accessible en ligne $<$ <ttp://www.sante.gov.mg/organigrammes/assets/uploads/files/ documents_officiels/cebdd-iem_document_2.pdf>

Robinson, B. E., Masuda, Y. J., Kelly, A., Holland, M. B., Bedford, C. et al. 2018. Incorporating land tenure security into conservation. Conservation Letters 11, 2 e12383. <https://doi.org/10.1111/conl.12383>

Robinson, L. W., Fava, F. P., Flintan, F. E., Frija, A., Louchaichi, M. \& Sircely, J. A. 2021 Community rangeland management approaches for land and ecosystem restoration: a biref review of ICARDA and ILRI research. CGSpace. CRP Livestock Briefs 7. Accessible en ligne <https://cgspace.cgiar.org/handle/10568/113869> 
Saïd, M. et Sibelet, N. 2004. Pour que la terre ne cache plus l'arbre : le foncier de I'arbre. Cahiers Agricultures 13, 6:510-515. Accessible en ligne $<$ https://revues.cirad.fr/index.php/cahiers-agricultures/article/view/ 30473/30233>

Scales, I. R. 2014. The future of biodiversity conservation and environmental management in Madagascar. lessons from the past and challenges ahead. In: Conservation and Environmental Management in Madagascar. I. R. Scales (ed), pp 342-360. Routledge, London. <https://doi.org/10.4324/9780203118313-28>

Slobodian, L., Vidal, A. \& Saint-Laurent, C. 2020. Policies that Support Forest Landscape Restoration: What they Look like and How they Work. IUCN, Gland, Switzerland. Accessible en ligne <https://portals.iucn.org/library/node/49261>

Solofondranohatra, C. L., Vorontsova, M. S., Hempson, G. P., Hackel, J., Cable, S., et al. 2020. Fire and grazing determined grasslands of central Madagascar represent ancient assemblages. Proceedings of the Royal Society B: Biological Sciences 287, 1927: 20200598. <https://doi.org/10.1098/rspb.2020.0598>

Stanturf, J. A, Kant, P., Lillesø, J.-P. B., Mansourian, S., Kleine, M., et al. 2015. Forest Landscape Restoration as a Key Component of Climate Change Mitigation and Adaptation. International Union of Forest Research Organizations Vienna, Austria. IUFRO World Series, Vol. 34. Accessible en ligne <https://www.iufro.org/uploads/media/ws34.pdf>

Stanturf, J. A., Mansourian, S. \& Kleine, M. 2017. Implementing Forest Landscape Restoration: A Practitioner's Guide. IUFRO. Accessible en ligne <https://www.iufro.org/science/special/spdc/netw/flr/flr/pract-guide/>

Weston, P., Hong, R., Kaboré, C. \& Kull., C. A. 2015. Farmer-managed natural regeneration enhances rural livelihoods in dryland west Africa. Environmental Management 55, 6: 1402-1417. <https://doi.org/10.1007/s00267-015-0469-1>

Wilson, S. J. \& Cagalanan, D. 2016. Governing restoration: Strategies, adaptations and innovations for tomorrow's forest landscapes. World Development Perspectives 4, 4: 11-15. <http://dx.doi.org/10.1016/j.wdp.2016.11.015>

Worku, T., Tripathi, S. K. \& Khare, D. 2018. Household level tree planting and its implication for environmental conservation in the Beressa Watershed of Ethiopia. Environmental Systems Research 6: 10.

$<$ https://doi.org/10.1186/s40068-017-0087-4>

Ykhanbai, H., Garg, R., Singh, A., Moiko, S., Beyene, C. E., et al. 2014. Conservation and 'Land grabbing' in Rangelands: Part of the Problem or Part of the Solution? International land coalition, Rangelands Series 5, iied Publications Library. Accessible en ligne <https://pubs.iied.org/g03853>

Zakout, W. \& White, A. 2019. Community Land Rights: An untapped Solution to Secure Climate, Biodiversity, and Development Goals. Accessible en ligne $<$ https://blogs.worldbank.org/sustainablecities/community-land-rights-untapped-solution-secure-climate-biodiversity-development-goals>
MATÉRIEL SUPPLÉMENTAIRE

La définition des forêts et leur régime juridique à Madagascar 